\title{
Non-targeted metabolomics of intestinal flora in seborrheic patients based on ultra-high performance liquid chromatography- quadrupole time-of-flight mass spectrometry (UHPLC-QTOF-MS) techniques
}

\author{
Lin Wang ${ }^{1}$, Qiaoyuan Ye $^{2}$, Jian Zhu ${ }^{1}$, Haobo Jiang ${ }^{1}$ \\ ${ }^{1}$ Department of Dermatology, Dongguan Tungwah Hospital, Dongguan, China; ${ }^{2}$ Department of Dermatology, The Second Clinical Medical \\ College, Guangdong Medical University, Dongguan, China \\ Contributions: (I) Conception and design: L Wang; (II) Administrative support: L Wang; (III) Provision of study materials or patients: All authors; (IV) \\ Collection and assembly of data: L Wang, J Zhu, H Jiang; (V) Data analysis and interpretation: L Wang; (VI) Manuscript writing: All authors; (VII) \\ Final approval of manuscript: All authors. \\ Correspondence to: Lin Wang. No. 1 Dongcheng East Road, Dongguan 523900, China. Email: wanglin0933@163.com.
}

Background To explore the role of intestinal flora in seborrhea, non-targeted metabolomics analysis was carried out.

Methods: Fecal samples were collected from 5 seborrheic patients and 5 healthy controls from October 2019 to April 2020. Ultra-high performance liquid chromatography-quadrupole time-of-flight mass spectrometry (UHPLC-QTOF-MS) was used to detect metabolic fingerprinting in feces samples, and highthroughput sequencing and bioinformatic analysis of $16 \mathrm{~S}$ rRNA for intestinal flora. The variable importance in projection (VIP) values of orthogonal partial least squares-discriminant analysis (OPLS-DA) and $\mathrm{P}$ values of univariate statistical analysis were used to determine the differential metabolites between the seborrhea group and the control group. The interaction between flora and metabolites was analyzed using several approaches.

Results: A total of 45 metabolites with significantly different intensities were found between the seborrhea group and the healthy control group. A positive correlation between flora and metabolites was found in 57 pairs and a negative correlation was found in 104 pairs. In addition, 11 metabolic pathways were significantly altered, including 4 amino acid metabolic pathways, 2 bile acid metabolic pathways, and 2 basic metabolic signaling pathways (ABC transporters pathway and mTOR signaling pathway). Central carbon metabolism in cancer, glutathione metabolism, protein digestion and absorption were also involved.

Conclusions: The occurrence of seborrhea may be related to changes in intestinal flora and metabolic pathways. There is a close association between seborrhea and amino acid metabolic pathways or ABC transporters.

Keywords: Ultra-high performance liquid chromatography-quadrupole time-of-flight mass spectrometry (UHPLC-QTOF-MS); Seborrhea; intestinal flora; metabolomics

Submitted Dec 02, 2020. Accepted for publication Apr 21, 2021.

doi: 10.21037/apm-21-331

View this article at: http://dx.doi.org/10.21037/apm-21-331 


\section{Introduction}

Seborrhea is caused by an excessive secretion of sebum by the sebaceous glands that are densely distributed on the forehead, nose, scalp and back. Inflammation of the skin, erythema, scales, or fungal infection can cause seborrheic dermatitis. Acinetobacter colonization with a secondary inflammation results in acne. Additionally, follicular worm infection and abnormal skin vascular function contribute to rosacea. Hence, seborrhea is generally considered as an independent disease, and is also an important symptom in the development of acne, rosacea, seborrheic dermatitis, seborrheic alopecia and other diseases. The causes of seborrhea may be related to high androgen levels, genetics, diet, metabolism, age, mental stress and other factors, and may also be significantly related to digestive tract dysfunction (1). Specifically, high androgen levels may be the key cause of seborrhea, and evidence has been provided that the rate of sebum secretion is higher in men than in women (2). The amount of sebum secretion in individuals overlaps exactly with the three peaks of hormone levels. The sebaceous glands are active in infants during the first 3 months of life under the influence of androgens from the mother, and gradually return to normal levels thereafter. During puberty, androgen levels rise again, sebaceous glands become hypertrophic, and sebum production increases. In women after menopause, sebum secretion decreases, as well as in men after the age of 70 years $(3,4)$

Numerous studies have shown that intestinal florametabolites are closely related to the development of several diseases, such as chronic kidney disease (5). Particularly, the interaction between intestinal flora and intestinal epithelial cells and neuronal immune cells can cause a series of endocrine and neuroimmune responses. Recently, it has been found that dysbiosis of the intestinal flora results in changes in androgen levels, disturbances in lipid metabolism, insulin resistance, and production of inflammatory factors, which are extremely associated with obesity, metabolic syndrome, reproductive and gastrointestinal diseases, and gynecological tumors (6-10). Therefore, we hypothesized that dysbiosis of the intestinal flora may also be inextricably linked to seborrhea.

Ultra-performance liquid chromatography/quadrupole time-of-flight mass spectrometry (UPLC-QTOF/ MS) technology is widely used in various fields. UPLCQTOF/MS based untargeted metabolomics analysis has been widely used in metabolomics research in recent years. This technique provides the maximum amount of information about the central carbon cycle metabolism, and can characterize and quantify as many metabolites in biological systems as possible, reflecting the total metabolite information to the maximum extent. It also has high sensitivity and separation for metabolites that are difficult to volatilize or have poor thermal stability. In this study, we performed a non-targeted metabolomic analysis of the gut flora of seborrheic patients and healthy individuals based on UHPLC-Q-TOF-MS technique, which is reported below.

We present the following article in accordance with the MDAR reporting checklist (available at http://dx.doi. org/10.21037/apm-21-331).

\section{Methods}

The study was conducted in accordance with the Declaration of Helsinki (as revised in 2013). The study was approved by medical ethics of Dongguan Tungwah Hospital and informed consent was taken from all individual participants.

\section{General patient information}

This study enrolled 10 healthy individuals (6 males and 4 females) and 10 patients with seborrheic dermatitis (6 males and 4 females) who were admitted to the Department of Dermatology of Donghua hospital (Dongguan, China) between October 2019 and April 2020. The median age of the patients and healthy subjects was $44.71 \pm 5.22$ years (range, 31-57 years) and 45.86 5 5.93 years (range, 3457 years), respectively. The study included the patients who exhibited the symptoms including facial oiliness, scalp oiliness, scalp scaling, and scalp erythema; were accompanied by seborrheic dermatitis, acne and folliculitis; originally intended to cooperate with the investigator in collecting fecal specimens; have lived in Dongguan for more than 2 years. All healthy participants, randomly selected with similar gender, age and body index as the enrolled patients, showed no seborrhea symptoms and settled in Dongguan for more than 2 years. Patients or healthy individuals who had taken glucocorticoids, antibiotics, probiotics, immunosuppressants, retinoids, herbal medicines within 6 months, were pregnant or lactating, smoked and drank, had malignancies or other serious internal diseases, had intestinal diseases, and had gastric and lower gastrointestinal surgery, were not included in this study. 


\section{Instruments and reagents}

An AB Triple TOF 5600/6600 mass spectrometer (AB SCIEX), an Agilent 1290 Infinity LC ultra-high pressure liquid chromatograph (Agilent), a cryogenic high-speed centrifuge (Eppendorf 5430R), and Illumina MiSeq platform (New England Biolabs, Ipswich, MA, USA) were used for analysis.

Chromatographic column: ACQUITY UPLC BEH Amide $1.7 \mu \mathrm{m}, 2.1 \mathrm{~mm} \times 100 \mathrm{~mm}$ column (Waters); ACQUITY UPLC HSS T3 $1.8 \mu \mathrm{m}, 2.1 \times 100 \mathrm{~mm}$ column (Waters), acetonitrile (Merck, 1499230-935), ammonium acetate (Sigma, 70221), QIAamp DNA Stool Mini Kit (Qiagen, Hilden, Germany), Agencourt AMPure XP beads (Beckman Coulter, Miami, FL, USA)

\section{Sample pretreatment}

Fecal samples $(80 \mathrm{mg})$, which were stored at $-80^{\circ} \mathrm{C}$, were thawed, mixed with $200 \mu \mathrm{L} \mathrm{H} \mathrm{H}_{2} \mathrm{O}$ and vortexed for $60 \mathrm{~s}$, followed by homogenization. Next, $800 \mu \mathrm{L}$ methanol acetonitrile solution $(1: 1, \mathrm{v} / \mathrm{v})$ was added to the mixture, followed by vortex for $60 \mathrm{~s}$ and low-temperature sonication for $30 \mathrm{~min}, 2$ times. The solution was then placed at $-20{ }^{\circ} \mathrm{C}$ for $1 \mathrm{~h}$ to precipitate the protein, and centrifuged at 14,000 rcf for $20 \mathrm{~min}$ at $4{ }^{\circ} \mathrm{C}$. Then the supernatant was collected and stored at $-80^{\circ} \mathrm{C}$.

\section{Gas chromatography-mass spectrometry (GC-MS)}

\section{Chromatography}

The samples were separated by an Agilent 1290 Infinity LC UHPLC column at $25^{\circ} \mathrm{C}$ with a flow rate of $0.3 \mathrm{~mL} / \mathrm{min}$ and mobile phase compound $\mathrm{A}$ : water $+25 \mathrm{mM}$ ammonium acetate $+25 \mathrm{mM}$ ammonia; and compound $\mathrm{B}$ : acetonitrile. Then the sample was eluted by using $95 \%$ ethanol for $0.5 \mathrm{~min}, 65 \%$ ethanol for $6.5 \mathrm{~min}, 40 \%$ ethanol for $1 \mathrm{~min}$, and $95 \%$ ethanol for $3 \mathrm{~min}$ in an autosampler at $4{ }^{\circ} \mathrm{C}$. The samples were treated in a random order to avoid the influence caused by the fluctuation of the instrument's detection signal. Quality control (QC) samples were used to evaluate the stability of the system and the reliability of experimental data.

\section{QTOF MS}

Electrospray ionization (ESI) positive and negative ion modes were used for detection. Following separation through UHPLC, the samples were analyzed using an Agilent 6550 mass spectrometer under the following conditions: gas temperature $250^{\circ} \mathrm{C}$, drying gas $16 \mathrm{~L} / \mathrm{min}$, nebulizer $20 \mathrm{psig}$, sheath gas temperature $400{ }^{\circ} \mathrm{C}$, sheath gas flow rate $12 \mathrm{~L} / \mathrm{min}$, Vcap $3000 \mathrm{~V}$, nozzle voltage: $0 \mathrm{~V}$, fragment $175 \mathrm{~V}$, mass range $50-1,200$, acquisition rate $4 \mathrm{~Hz}$, cycle time $250 \mathrm{~ms}$.

\section{Statistical analysis}

Original data were converted into the mzXML format by ProteoWizard, and then the XCMS program was used for peak alignment, retention time correction, and peak area extraction. The metabolite structure was identified with accurate mass matching $(<25 \mathrm{ppm})$, secondary spectrum matching, and detected by the laboratory's self-built database.

After being preprocessed by Pareto-scaling, data were analyzed by multi-dimensional statistical analyses, comprising of unsupervised principal component analysis (PCA), supervised partial least squares discriminant analysis (PLS-DA), and orthogonal partial least squares discriminant analysis (OPLS-DA). One-dimensional statistical analyses were performed, including a Student's $t$-test and variation analysis, and volcano maps using $\mathrm{R}$ software.

\section{High-throughput sequencing and bioinformatic analysis of $16 S r R N A$}

DNA was extracted using QIAamp DNA Stool Mini Kit following the manufacturer's description. Then 6S V3-V4: 341F-806R, 18S V9: 1380F-1510R, ITS1: ITS1F- ITS2R. 16S/18S rRNA genes were amplified used the specific primer (Table 1) with the barcode, followed by confirmation by PCR and purification with Agencourt AMPure XP beads. Next, the $16 \mathrm{~S}$ rRNA sequencing was performed with an Illumina MiSeq platform. The obtained reads were analyzed using the QIIME 1.9.1 package after a quality filtering and removal of ambiguous nucleotides. Amplicon sequences were clustered into operational taxonomic units and used to characterize microbiota diversity. Sequences not aligning the references were excluded.

\section{$16 S$ rRNA non-targeted metabolic analysis}

The interactions between flora and metabolites were analyzed using multiple approaches. The correlation coefficients between the flora and significant differential metabolites were detected by Spearman's correlation analysis, matrix heat maps, correlation network analysis, and hierarchical clustering using $\mathrm{R}$ language and Cytoscape software. 


\section{Results}

\section{Quality analysis}

When comparing the positive ion and negative ion modes of UHPLC-QTOF-MS, we found their response intensity and most retention time of peaks overlapped (Figures 1 and 2).

Table 1 Specific primers used for PCR

\begin{tabular}{ll}
\hline Primer name & Sequences \\
\hline 16S V3-V4 & \\
$341 \mathrm{~F}$ & 5'-CCTAYGGGRBGCASCAG-3' \\
$806 \mathrm{R}$ & 5'-GGACTACNNGGGTATCTAAT-3' \\
18S V9 & \\
1380F & 5'-GCCTCCCTCGCGCCATCAGXXXXXCCCTGC \\
& CHTTTGTACACAC-3' \\
1510R & 5'-GCCTTGCCAGCCCGCTCAGCCTTCYGCAG \\
& GTCACCTAC-3' \\
ITS1 & \\
ITS1F & 5'-CTTGGTCATTTAGAGGAAGTAA-3' \\
ITS2R & 5'-GCTGCGTTCTTCATCGATGC-3' \\
\hline
\end{tabular}

This indicated that the variation due to instrument error was minor, the system was stable, and the data were stable and reliable. The differences in metabolic profiles were reflections of biological differences between samples. The ion peaks of metabolites were isolated using XCMS software and calculated. The peaks of all the experimental samples and QC samples were presented in the PCA model through Pareto-scaling. As revealed in Figure 3, the QC samples in the positive and negative ion modes were closely clustered together, indicating good repeatability of this experiment. The correlation coefficients all exceeded 0.9 , indicating a good correlation between the 2 modes (Figure 4).

\section{Identification of differential metabolites}

OPLS-DA was performed to model the relationship between metabolite expression and sample category in negative and positive ion modes (Figures 5 and 6 ). It was clear that samples of the healthy control group were all distributed in the right area, and the seborrhea group samples were distributed in the left area, which indicated a significant difference in composition between the 2 groups. The volcano plot of the univariate statistical analysis

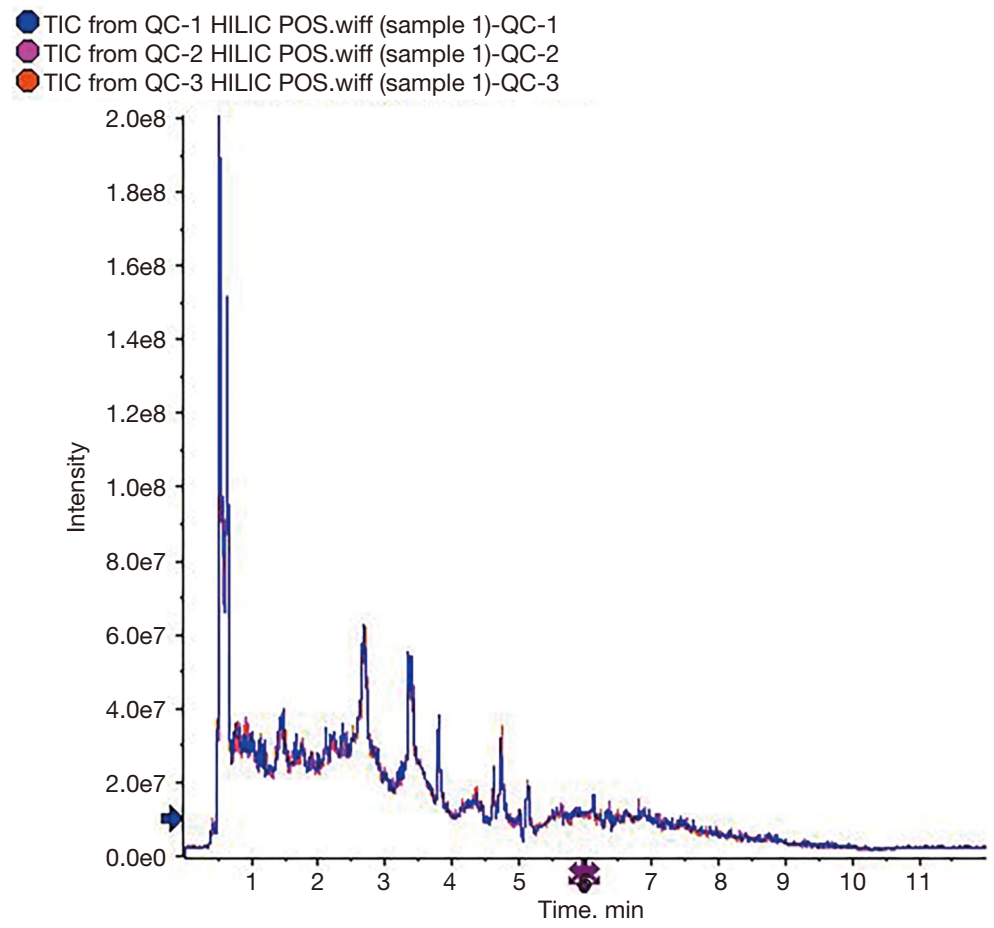

Figure 1 The overlapped total ion chromatography (TIC) of quality control (QC) samples in positive ion mode. The arrows and asterisks show the response intensity and retention time of each peak, respectively. 


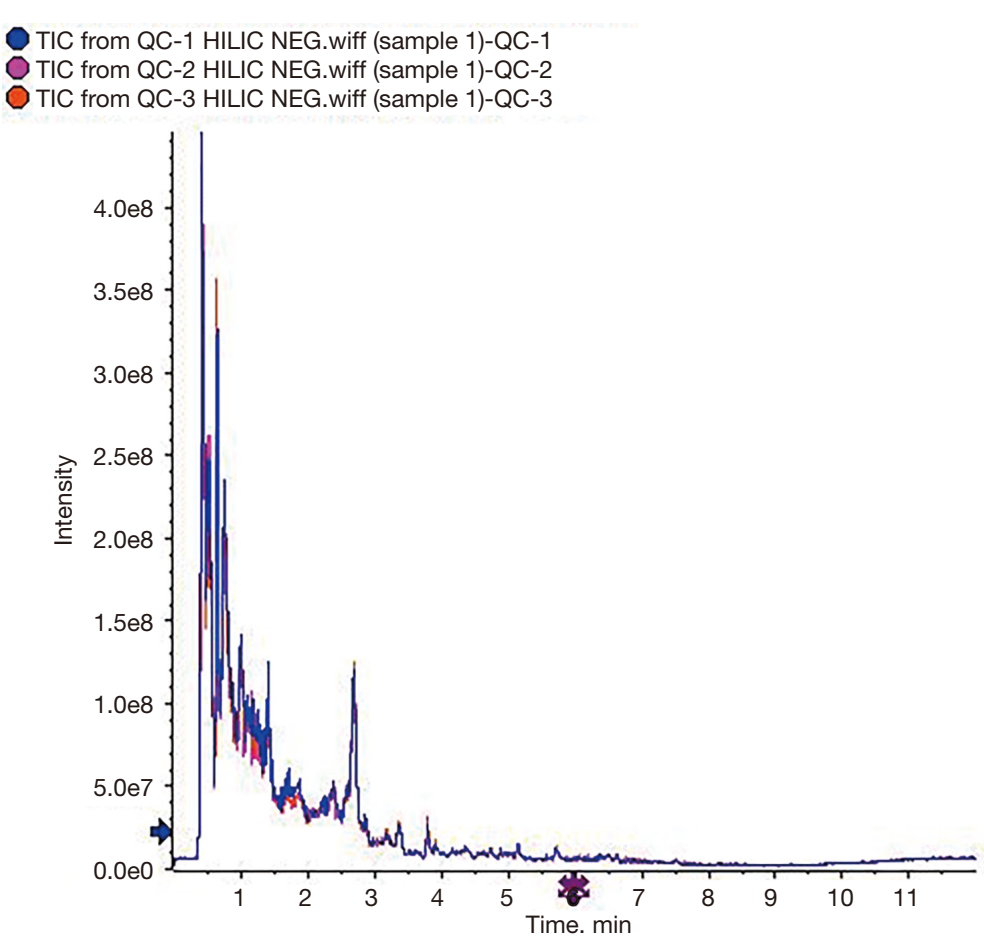

Figure 2 The overlapped total ion chromatography (TIC) of quality control (QC) samples in negative ion mode. The arrows and asterisks show the response intensity and retention time of each peak, respectively.

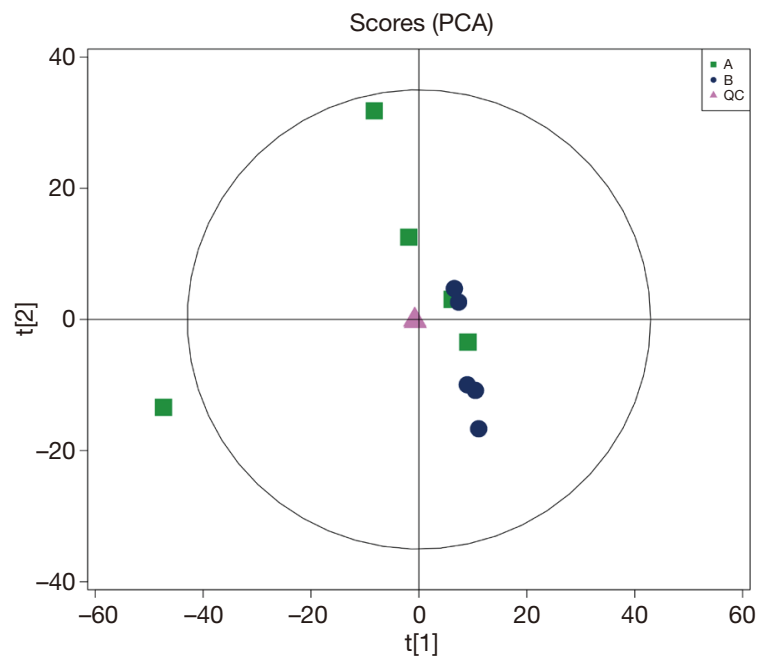

Figure 3 Principal component analysis (PCA) score plot of quality control (QC) samples in positive and negative ion modes.

screened the differential metabolites (red), with fold change (FC) $>1.5$ and $\mathrm{P}$ value $<0.05$ in the positive ion mode (Figure 7). Furthermore, 45 differential metabolites were screened by multivariate statistical analysis with variable importance in projection (VIP) $>1$ and univariate statistical analysis with $\mathrm{P}<0.05$, and some of the metabolites are shown in Table 2.

\section{Bioinformatics analysis of differential metabolites}

In this study, hierarchical clustering was carried out to determine the significant differential metabolites of groups in positive ion mode (Figure 8). Interestingly, we found that the seborrhea group and the healthy control group were clustered in the same cluster. Metabolites in the same cluster have similar expression patterns at close reaction steps during metabolic processes, indicating that the candidate metabolites selected in this study were reasonable and accurate. In addition, there was a significant regulatory relationship between the metabolites detected in a positive ion mode (Figure 9).

\section{Analysis of differential Kyoto Encyclopedia of Genes and Genomes (KEGG) patbways}

KEGG pathway enrichment analysis of differentially expressed metabolites from the patients with seborrhea 


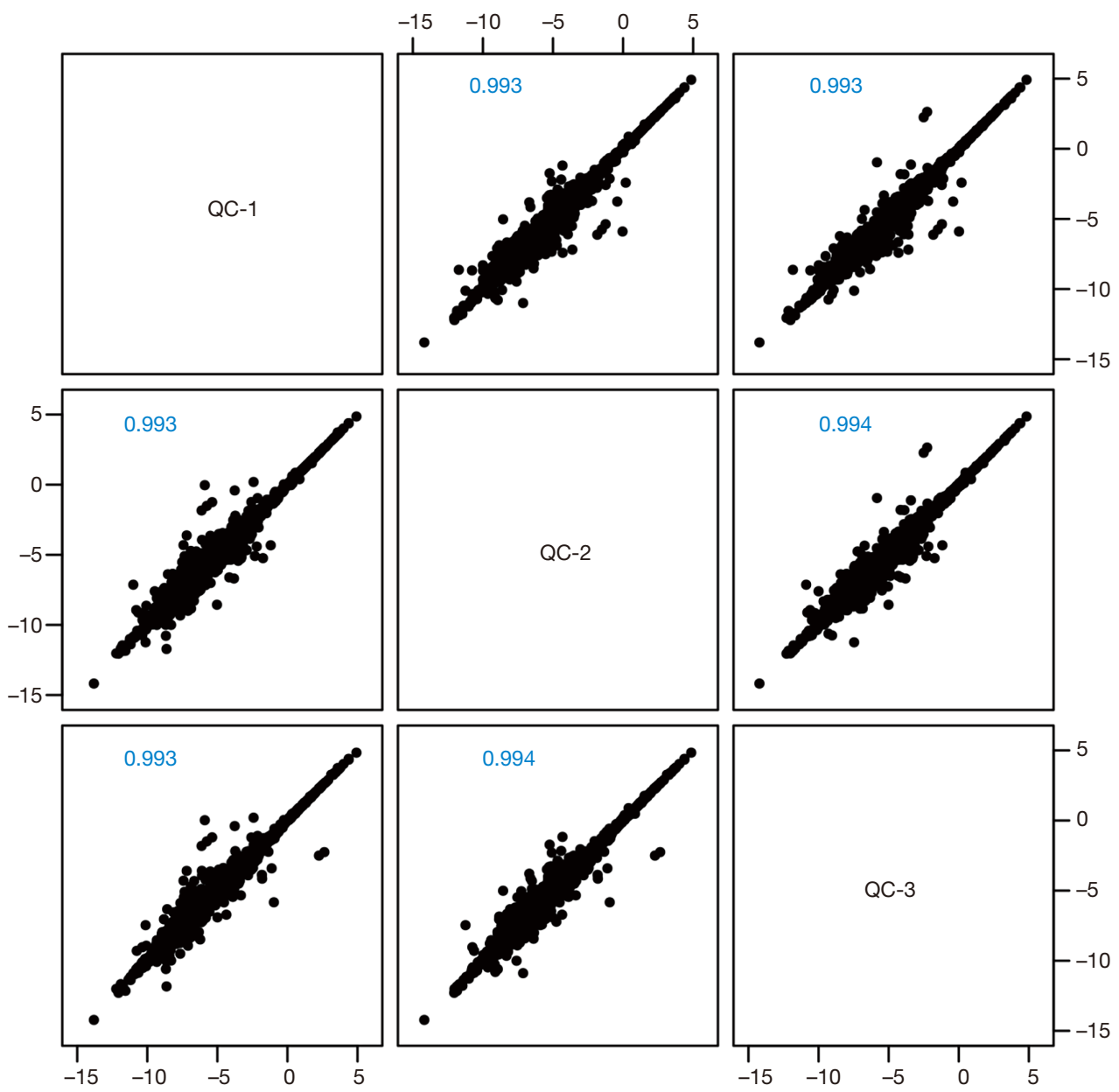

Figure 4 Correlation analysis between quality control (QC) samples in positive and negative ion modes.

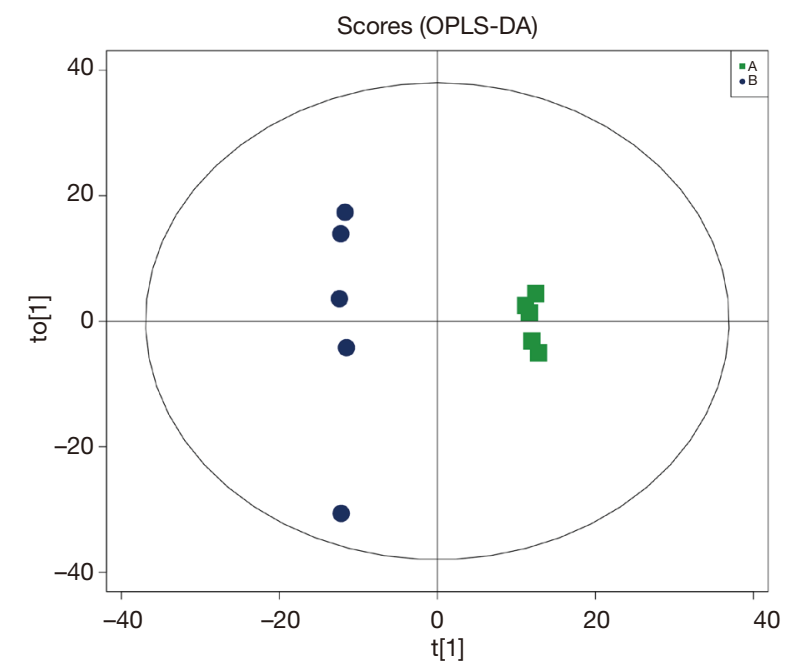

Figure 5 Orthogonal partial least squares discriminant analysis (OPLS-DA) of fecal samples in the negative and positive ion modes.

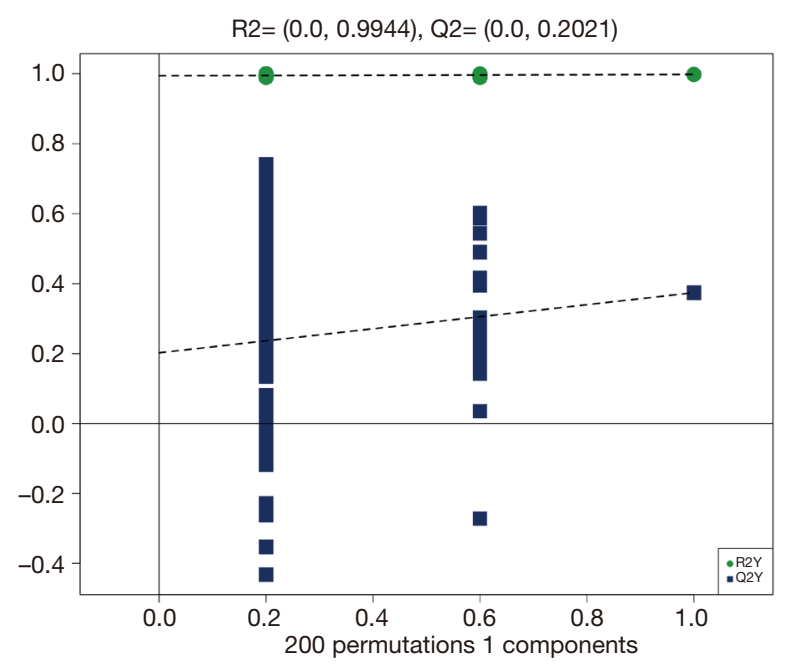

Figure 6 Orthogonal partial least squares discriminant analysis (OPLS-DA) permutation test in the negative and positive ion modes. 


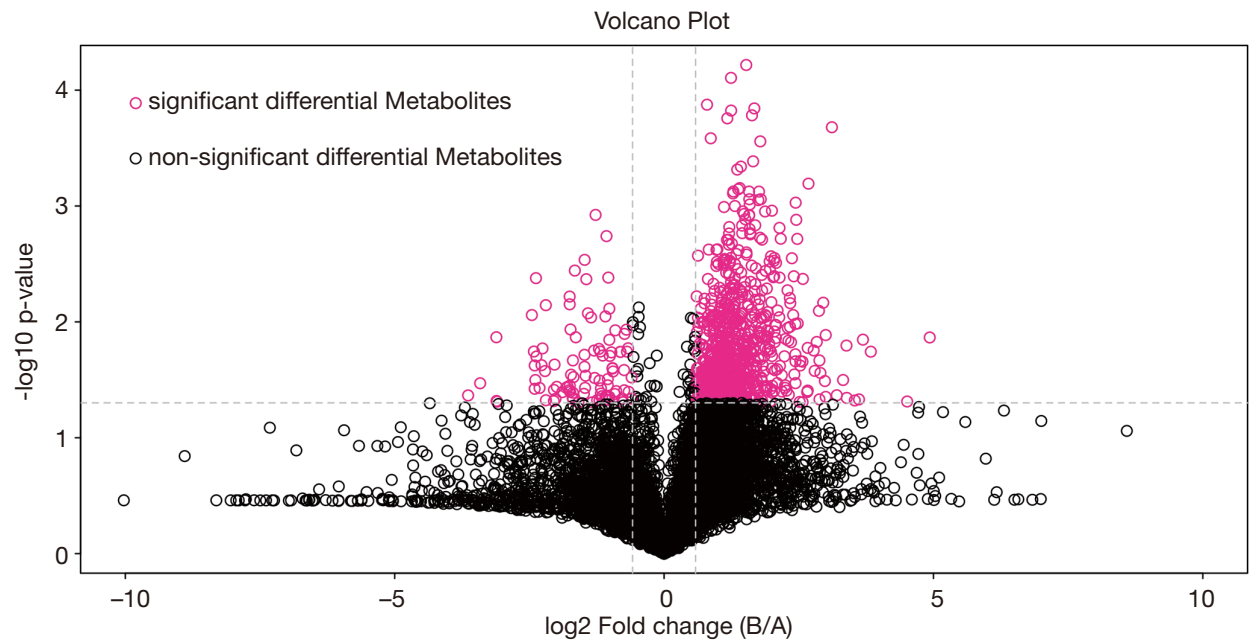

Figure 7 Volcano plot showing the differentially expressed metabolites. Fold change (FC) $>1.5$ : metabolites in seborrheic samples (B) were significantly increased compared to control samples (A). FC $<1.5$ : metabolites in seborrheic samples (B) were significantly decreased compared to control samples (A).

Table 2 Differential metabolites identified by positive ion mode

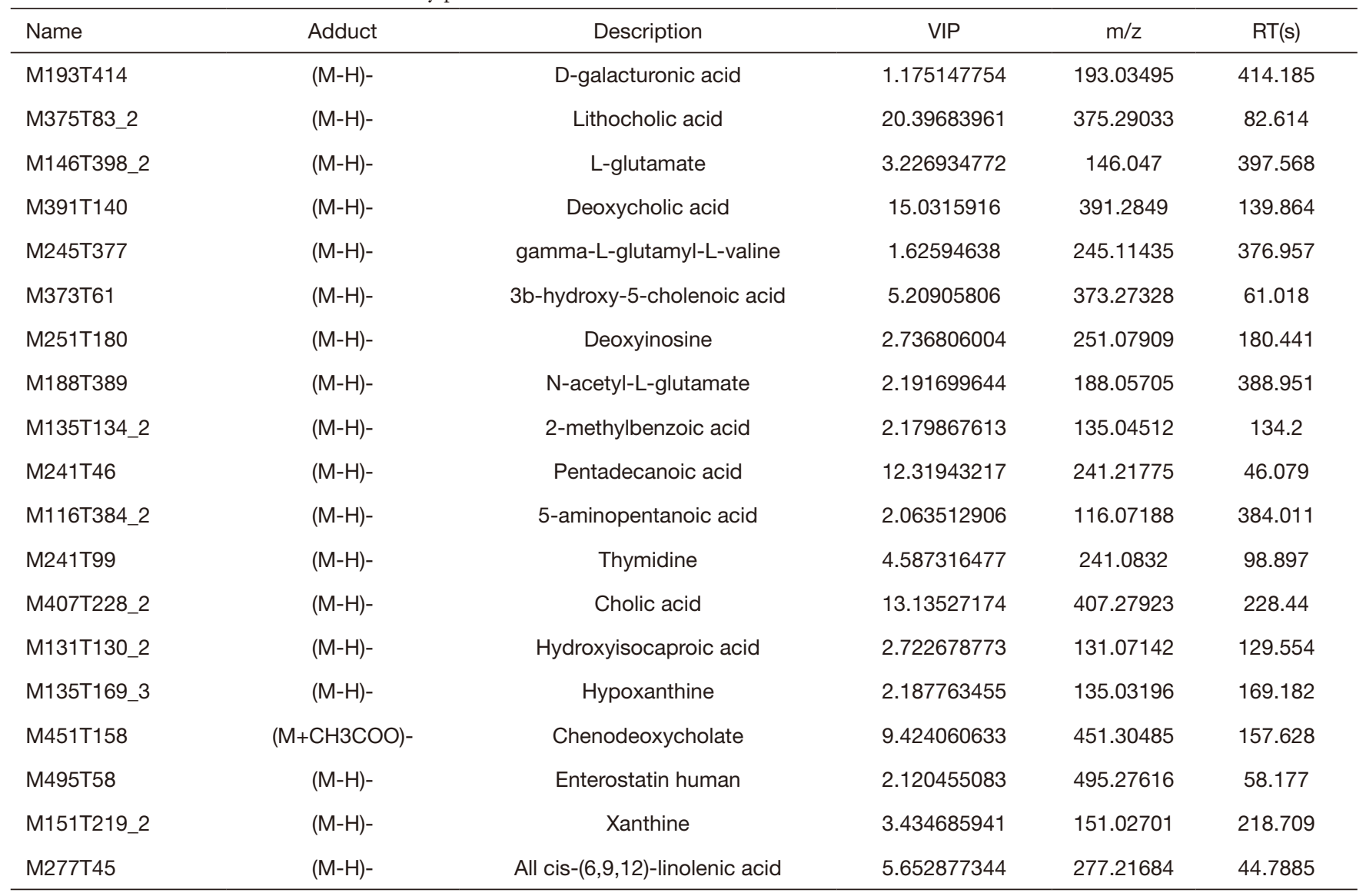

VIP, variable importance in projection; RT, retention time; M, molecule; m/z, mass-to-charge ratio. 


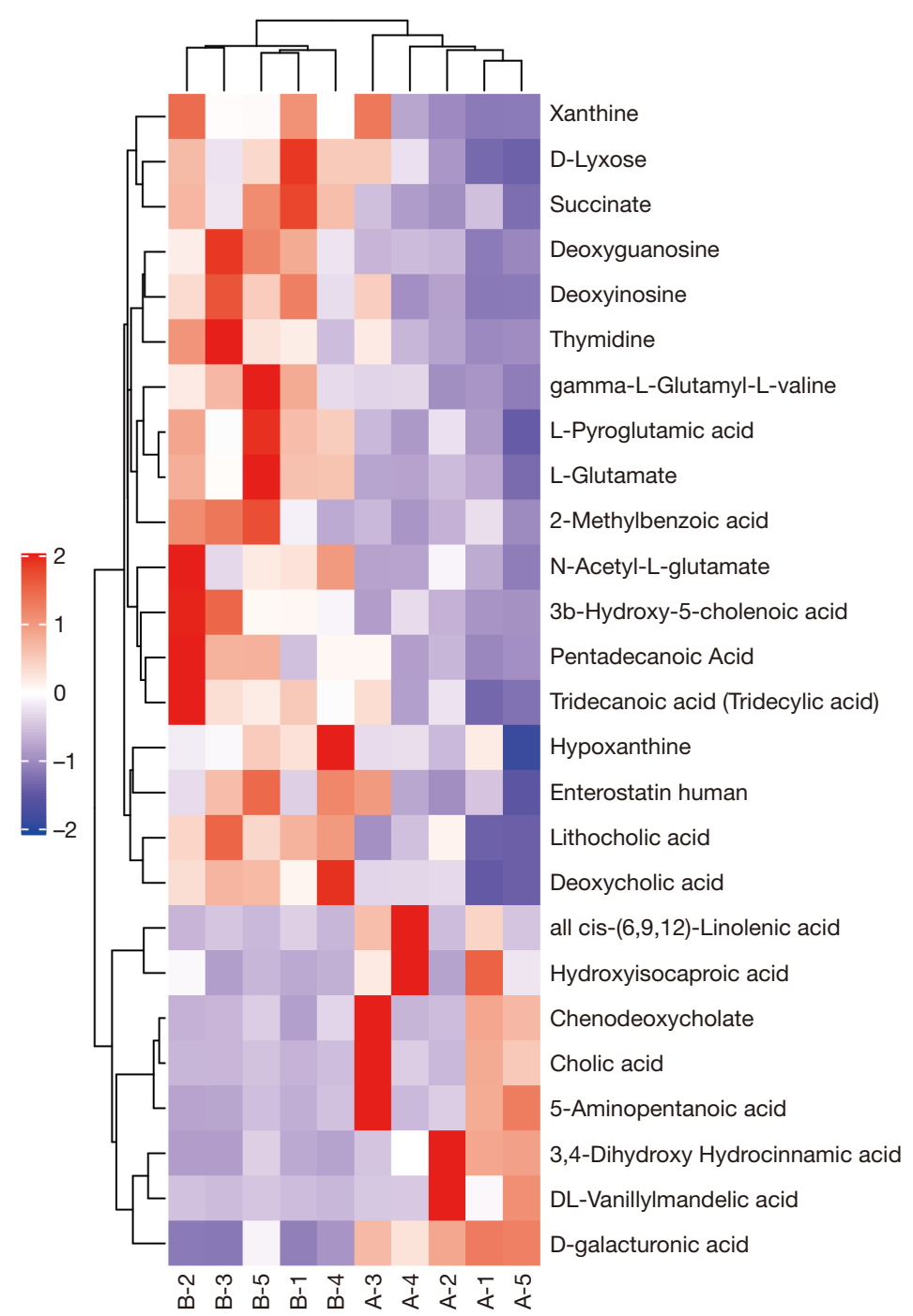

Figure 8 Significant differential metabolites as determined by hierarchical clustering. Group A: control samples; Group B: seborrheic samples.

or healthy individuals was performed using Fisher's exact test (Figure 10). The results showed significant differences in important pathways, including ATP-binding cassette transporters ( $\mathrm{ABC}$ transporters), purine metabolism, arginine and proline metabolism, arginine biosynthesis, and central carbon metabolism in cancer (Figure 11).

\section{S non-targeted metabolism joint analysis}

Experimental samples were analyzed using 16S rRNA amplicon sequencing analysis, and 15 differential flora and their relative abundance were also analyzed [linear discriminant analysis (LDA) effect size (LefSe) $>2$ and $\mathrm{P}$ value $<0.05]$. A total of 45 differential metabolites were found (VIP $>1$ and $\mathrm{P}$ value of $t$-test $<0.05$ ). The interaction between these flora and metabolites was analyzed by correlation coefficient analysis, matrix heat map analysis, cluster analysis, correlation network analysis, and scatter point graph analysis. We displayed the correlation between differential flora and metabolites in the form of a correlation coefficient matrix heat map (Figure 12). It was found that the 2 groups of samples varied in terms of metabolomics and bacterial diversity. Spearman's statistical analysis indicated a positive correlation in 57 pairs of flora-metabolites and a 


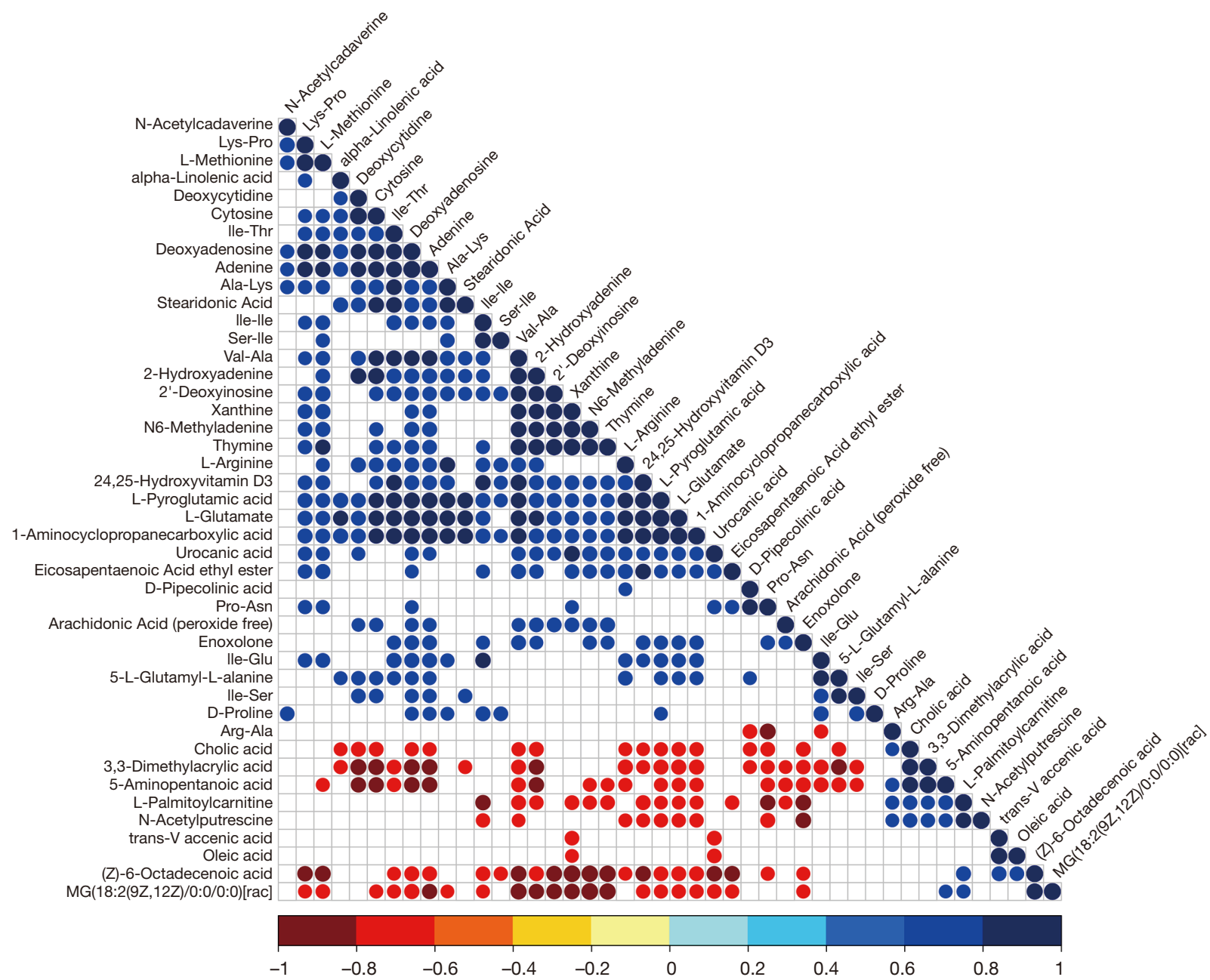

Figure 9 Correlation analysis of differential expression metabolites from seborrheic samples and control samples.

negative correlation in 104 pairs. Furthermore, Spearman's correlation network screened significant differential metabolites and flora with correlation coefficient absolute values between $[0.5,1]$ and $\mathrm{P}$ value $<0.05$ (Figure 13).

\section{Discussion}

In this paper, we performed an analysis of the metabolic components of fecal samples from the seborrhea group and the healthy control group, and found 45 significant differential metabolites. They mainly included amino acids and their derivatives, followed by bile acids, bile salts and their derivatives, bases and their derivatives, and a small amount of fatty acids. Results from 16S-RNA sequencing showed that 15 species of bacteria were differently accumulated in seborrhea patients relative to healthy individuals, with Fusobacteria, Bacteroidaceae, Bacteroides and Bromii decreased, and Enterobacteriaceae, Faecalibacterium, Blautia, Prausnitzii, Gnavus and Coli increased. In terms of metabolic pathways, the results showed that 11 metabolic pathways were significantly altered, including 4 amino acid metabolic pathways, 2 bile acid metabolic pathways, and 2 basic metabolic signaling pathways (ABC transporters pathway and mTOR signaling pathway). Central carbon metabolism in cancer, glutathione metabolism, protein digestion and absorption were also involved. Among them, the most closely related metabolic pathways included biosynthesis of amino acids, ABC transporters, bile secretion and arginine and proline metabolism.

Four of the 11 signaling pathways were predicted to 


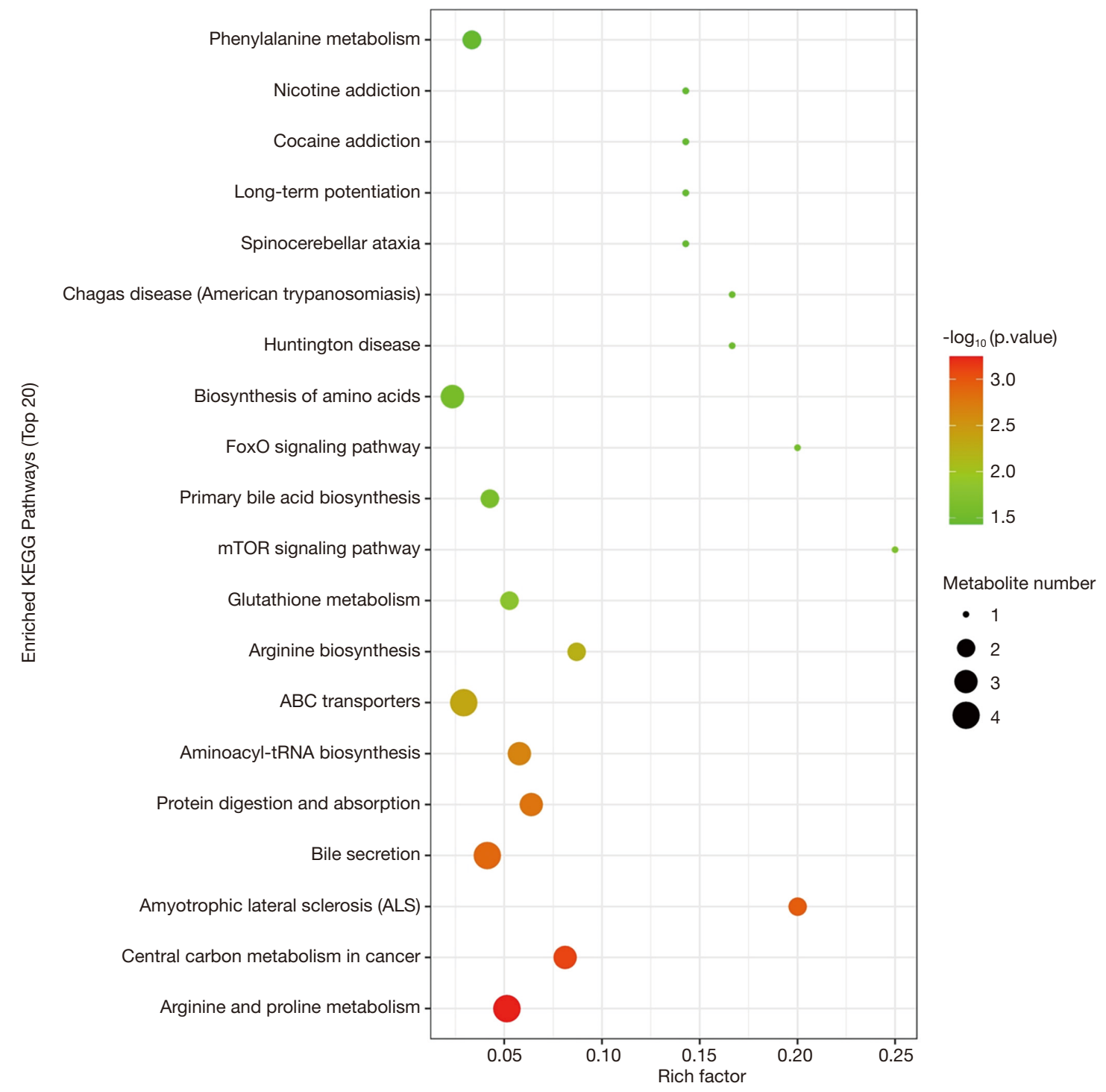

Figure 10 Kyoto Encyclopedia of Genes and Genomes (KEGG) pathway enrichment analysis.

participate in regulating amino acid metabolism. Besides, the highest percentage of abnormal metabolites were also amino acid, suggesting a close link between seborrhea and amino acid metabolism. In vitro studies have shown that Toll receptor 2 and Toll receptor 4 in keratinforming cells of the epidermis recognize Acinetobacter and initiate downstream natural immunity through inducing gene expression, and thus result in acne. Mechanically, changes in arginine and glycine impair the regulation of downstream immune signaling and gene expression, which ultimately mediates acne $(11,12)$. Ruiz et al. found that a key link in insulin resistance caused by intestinal flora disorders is the activation of Toll receptors (TLRs) signaling pathway, which could explain the high prevalence of insulin resistance in patients with severe acne (13). It was suggested that acne patients have a high prevalence of psychological disorders of depression and anxiety (14), while another study has shown that abnormal amino acid metabolism is closely related to depression (15). According to our results, we proposed that intestinal flora disorders cause abnormal amino acid metabolism, which leads to depression. Because sebaceous gland cells have multiple receptors, including adrenocorticotropin-releasing hormone, androgens, and several neuropeptides, then it 


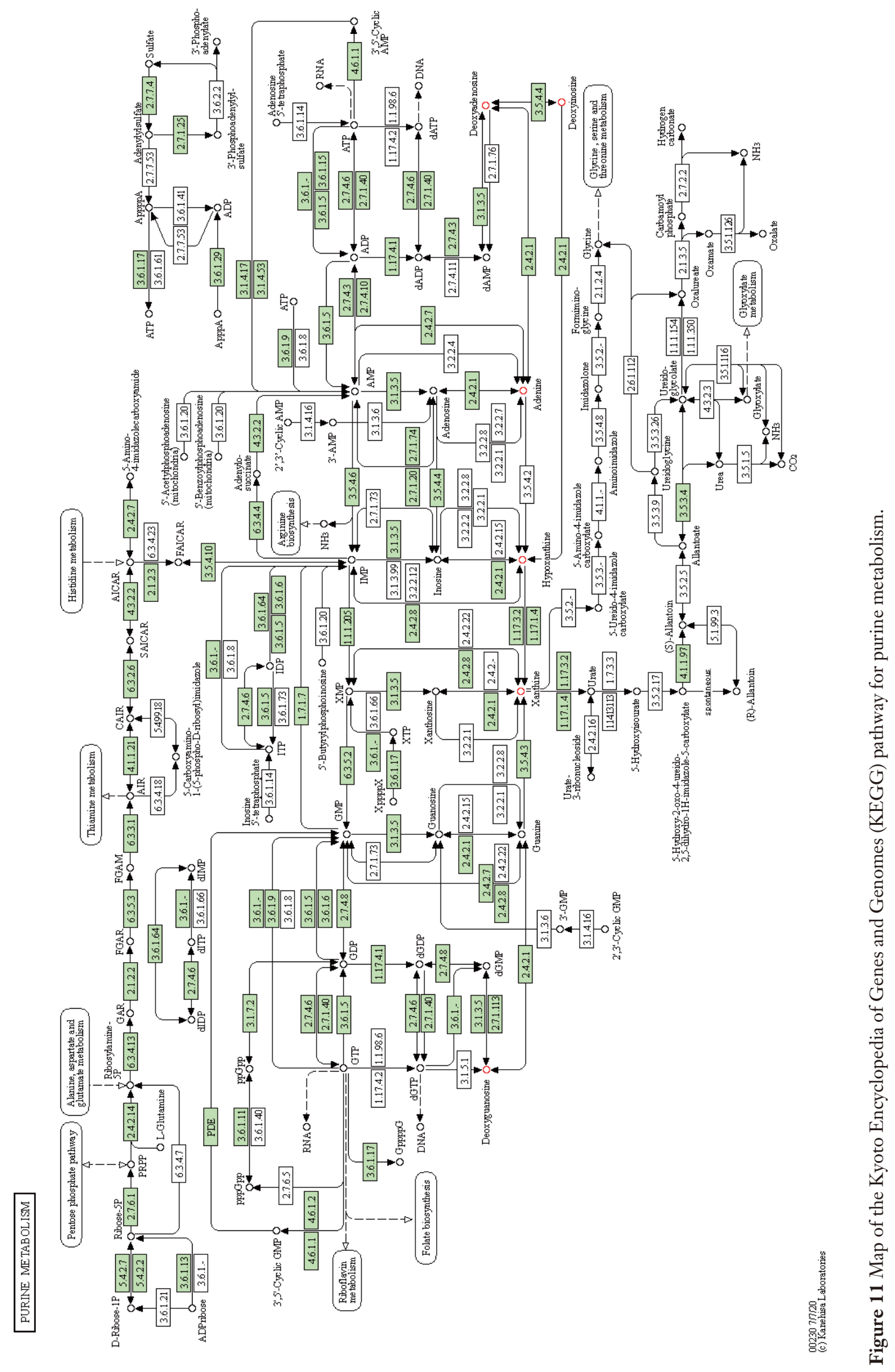




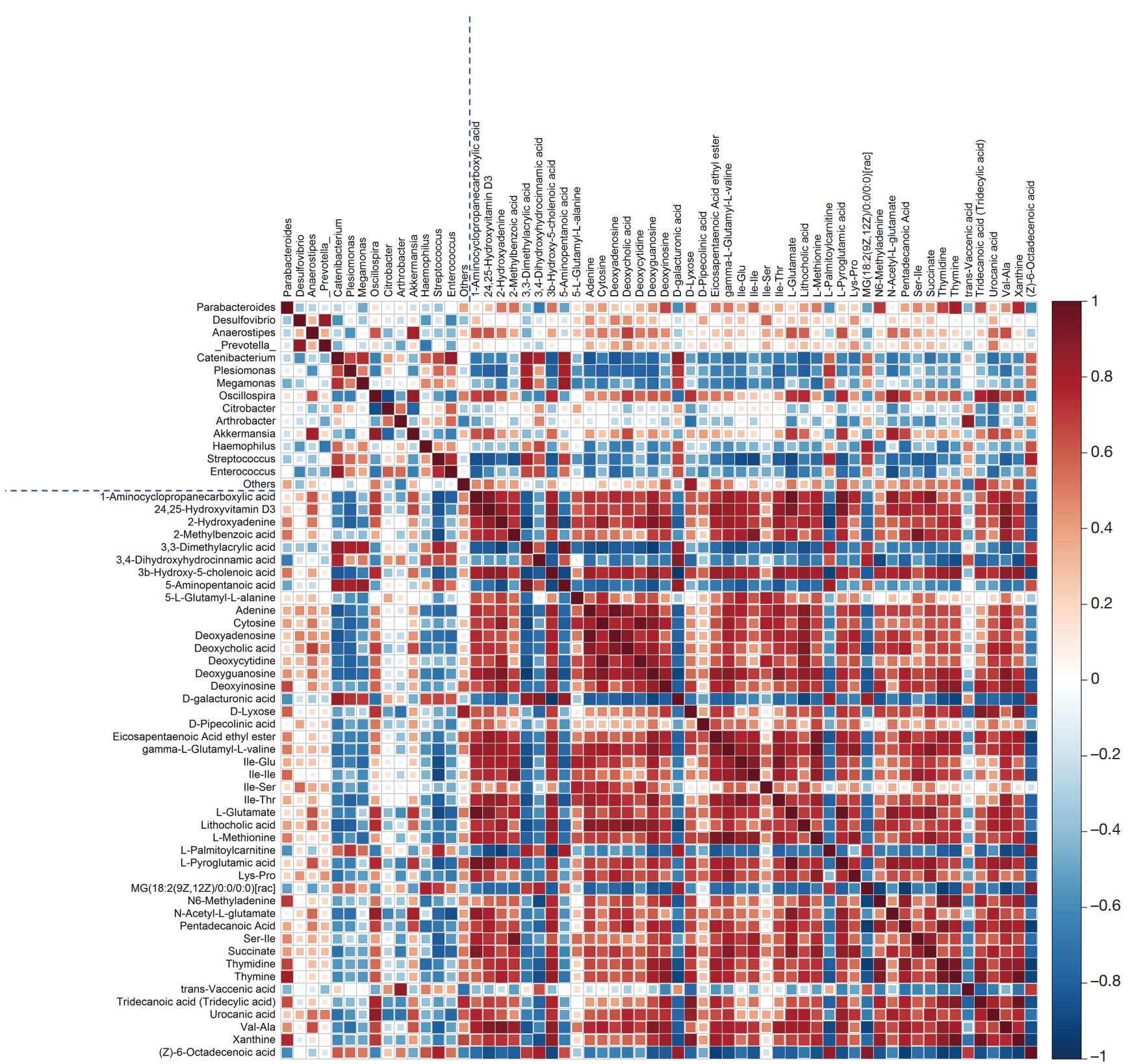

Figure 12 Heatmap of the correlation coefficient matrix for the differentially expressed metabolites in fecal samples from seborrheic patients and healthy individuals.

may be that the stress response acts through hormone levels and neurotransmitters, thus affecting the synthetic and secretory functions of sebaceous glands (16-18).

It is believed that disorders of the intestinal flora lead to abnormal functioning of $\mathrm{ABC}$ transporters, which modulate lipid metabolism, thereby driving seborrhearelated diseases. The primary thought was that the ABC transporters in bacteria were closely related to bacterial nutrient uptake. Studies in the 1990s found that ABC transporters are not only ubiquitous in organisms, but are also involved in various physiological and biochemical processes. $\mathrm{ABC}$ transporters are now considered to transport a variety of substrates, from simple ions and polypeptides with polar, amphoteric, and hydrophobic 


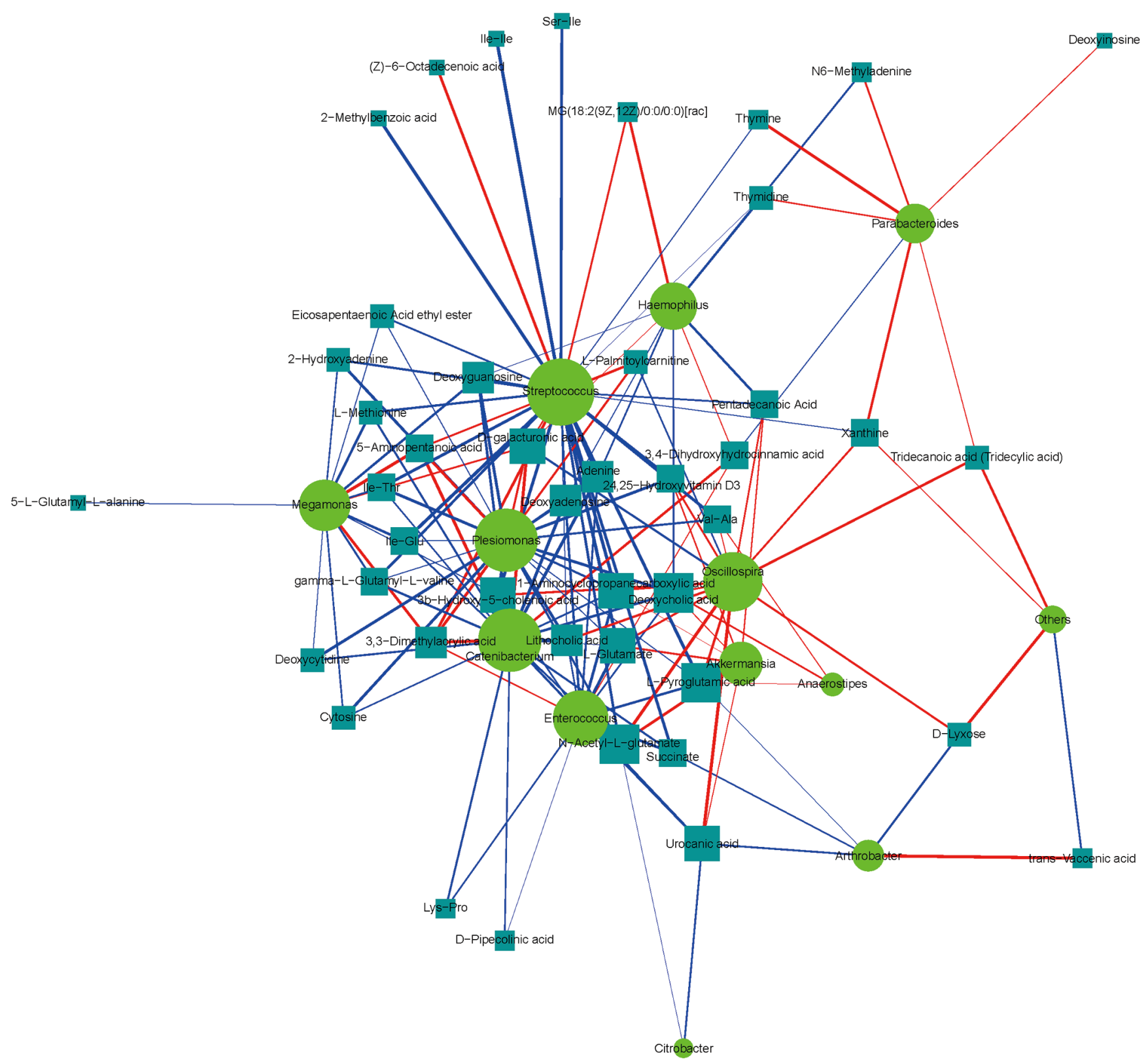

Figure 13 Correlation network analysis of the differentially expressed metabolites in fecal samples from seborrheic patients and healthy individuals.

organic molecules, to complex lipids and small proteins. $\mathrm{ABC}$ transporter dysfunction can cause many diseases, such as familial high-density lipoprotein deficiency, Alzheimer's disease, immunodeficiency, diabetes, coronary heart disease and cholelithiasis $(19,20)$. In this study, decreased content of $\mathrm{ABC}$ transporters was observed in the intestinal flora of the seborrhea group. It is speculated that disorders of the intestinal flora might cause abnormalities of the $\mathrm{ABC}$ transporters through regulation of lipid metabolism.

Abnormal purine and amino acid metabolism can cause various diseases, including gout, immune system disorders (such as immunodeficiency syndrome), blood disorders, kidney diseases, nervous system dysfunction, and depression $(15,21)$. It has been shown that diet is an important factor 
in the development of seborrheic diseases. In our study, patients in the seborrhea group had a high-fat and highcholesterol diet. Here, we proved that abnormal metabolism is closely related to the onset of seborrhea, and a high-fat and high-cholesterol diet-induced metabolic disorder may be associated with seborrhea diseases.

There are many diverse metabolites of the intestinal flora, and the present study only focused on some of them. In conclusion, our findings suggest that metabolites related to the gut microbiota may become biomarkers for exploring its roles in seborrhea. Our findings may contribute to the development of microbiota-based tools for the diagnosis, prevention, and treatment of seborrheic diseases.

\section{Acknowledgments}

Funding: This work was supported by the General Project of Dongguan Social Science and Technology Development (Project No. 2018507150461226)

\section{Footnote}

Reporting Checklist: The authors have completed the MDAR reporting checklist. Available at http://dx.doi.org/10.21037/ apm-21-331

Data Sharing Statement: Available at http://dx.doi. org/10.21037/apm-21-331

Conflicts of Interest: All authors have completed the ICMJE uniform disclosure form (available at http://dx.doi. org/10.21037/apm-21-331). The authors have no conflicts of interest to declare.

Ethical Statement: The authors are accountable for all aspects of the work in ensuring that questions related to the accuracy or integrity of any part of the work are appropriately investigated and resolved. The study was conducted in accordance with the Declaration of Helsinki (as revised in 2013). The study was approved by medical ethics of Dongguan Tungwah Hospital and informed consent was taken from all individual participants.

Open Access Statement: This is an Open Access article distributed in accordance with the Creative Commons Attribution-NonCommercial-NoDerivs 4.0 International License (CC BY-NC-ND 4.0), which permits the noncommercial replication and distribution of the article with the strict proviso that no changes or edits are made and the original work is properly cited (including links to both the formal publication through the relevant DOI and the license). See: https://creativecommons.org/licenses/by-nc-nd/4.0/.

\section{References}

1. Deng $\mathrm{Y}$, Wang H, Zhou J, et al. Patients with Acne Vulgaris Have a Distinct Gut Microbiota in Comparsion with Healthy Controls. Acta Derm Venereol 2018;98:783-90.

2. Ro BI, Dawson TL. The role of sebaceous gland activity and scalp microfloral metabolism in the etiology of seborrheic dermatitis and dandruff. J Investig Dermatol Symp Proc 2005;10:194-7.

3. Borda LJ, Wikramanayake TC. Seborrheic Dermatitis and Dandruff: A Comprehensive Review. J Clin Investig Dermatol 2015;3:10.13188/2373-1044.1000019.

4. Niemann C, Horsley V. Development and homeostasis of the sebaceous gland. Semin Cell Dev Biol 2012;23:928-36.

5. Roberts IS. Pathology of IgA nephropathy. Nat Rev Nephrol 2014;10:445-54.

6. Chong R, Xiao X. Intestinal microbiota and lipid metabolic disorders. Chinese Journal of Clinicians 2016;10:1053-5.

7. Liu R, Zhang C, Shi Y, et al. Dysbiosis of Gut Microbiota Associated with Clinical Parameters in Polycystic Ovary Syndrome. Front Microbiol 2017;8:324.

8. Yu J, Li H, Hu Q. Research on Gut Microbiota Diversity in Patients with Ulcerative Colitis by High-throughput Sequencing. Acta Med Univ Sci Technol Huazhong 2018;47:460-5.

9. Bäckhed F, Ding H, Wang T, et al. The gut microbiota as an environmental factor that regulates fat storage. Proc Natl Acad Sci U S A 2004;101:15718-23.

10. Qiao Y. The effect of oxidative stress induced by high fat diet on changes of gut microbiota and inflammation in mice 2014. Jiangnan University 2014:26-32.

11. Brown RA, Gralewski JH, Razonable RR. The R753Q polymorphism abrogates toll-like receptor 2 signaling in response to human cytomegalovirus. Clin Infect Dis 2009;49:e96-9.

12. Brown RA, Gralewski JH, Eid AJ, et al. R753Q singlenucleotide polymorphism impairs toll-like receptor 2 recognition of hepatitis $\mathrm{C}$ virus core and nonstructural 3 proteins. Transplantation 2010;89:811-5.

13. Ruiz AG, Casafont F, Crespo J, et al. Lipopolysaccharidebinding protein plasma levels and liver TNF-alpha gene expression in obese patients: evidence for the potential 
role of endotoxin in the pathogenesis of non-alcoholic steatohepatitis. Obes Surg 2007;17:1374-80.

14. Gupta MA, Pur DR, Vujcic B, et al. Suicidal behaviors in the dermatology patient. Clin Dermatol 2017;35:302-11.

15. Lach G, Schellekens H, Dinan TG, et al. Anxiety, Depression, and the Microbiome: A Role for Gut Peptides. Neurotherapeutics 2018;15:36-59.

16. Dixon LJ, Witcraft SM, McCowan NK, et al. Stress and skin disease quality of life: the moderating role of anxiety sensitivity social concerns. Br J Dermatol 2018;178:951-7.

17. Dreno B, Bagatin E, Blume-Peytavi U, et al. Female type of adult acne: Physiological and psychological considerations and management. J Dtsch Dermatol Ges 2018;16:1185-94.

18. Slominski AT, Zmijewski MA, Zbytek B, et al. Key role

Cite this article as: Wang L, Ye Q, Zhu J, Jiang H. Nontargeted metabolomics of intestinal flora in seborrheic patients based on ultra-high performance liquid chromatographyquadrupole time-of-flight mass spectrometry (UHPLC-QTOFMS) techniques. Ann Palliat Med 2021;10(4):4354-4368. doi: 10.21037/apm-21-331 of CRF in the skin stress response system. Endocr Rev 2013;34:827-84.

19. Tarling EJ, de Aguiar Vallim TQ, Edwards PA. Role of ABC transporters in lipid transport and human disease. Trends Endocrinol Metab 2013;24:342-50.

20. De Roeck A, Van Broeckhoven C, Sleegers K. The role of ABCA7 in Alzheimer's disease: evidence from genomics, transcriptomics and methylomics. Acta Neuropathol 2019;138:201-20.

21. Rock KL, Kataoka H, Lai JJ. Uric acid as a danger signal in gout and its comorbidities. Nat Rev Rheumatol 2013;9:13-23.

(English Language Editor: C. Betlazar-Maseh) 